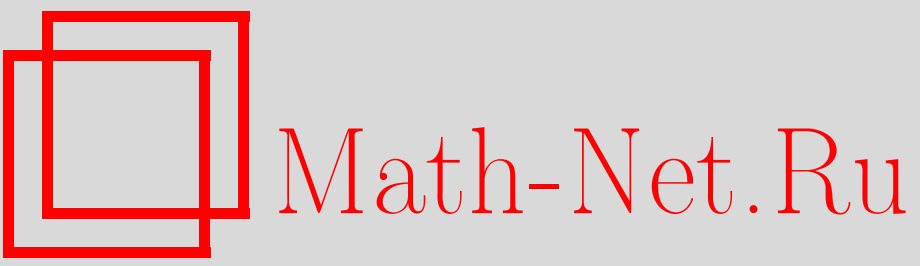

О. А. Малафеев, А. П. Парфенов, Компромиссное решение в модели экономики с заменой оборудования, Итоги науки и техн. Сер. Соврем. мат. и ее прил. Темат. обз., 2019, том 171, 125-139

DOI: https://doi.org/10.36535/0233-6723-2019-171-125-139

Использование Общероссийского математического портала Math-Net.Ru подразумевает, что вы прочитали и согласны с пользовательским соглашением

http: //www. mathnet.ru/rus/agreement

Параметры загрузки:

IP : 18.234 .156 .22

26 апреля 2023 г., 18:01:55 


\title{
КОМПРОМИССНОЕ РЕШЕНИЕ В МОДЕЛИ ЭКОНОМИКИ С ЗАМЕНОЙ ОБОРУДОВАНИЯ
}

\author{
(C) 2019 г. \\ О. А. МАЛАФЕЕВ, А. П. ПАРФЕНОВ
}

\begin{abstract}
АннотАция. Формализована задача оптимальной стратегии замены оборудования в отрасли экономики при наличии ценовых ожиданий и ожиданий технического прогресса, нейтрального по Харроду. Нужно определить стратегию покупки и списания оборудования в каждый момент времени, минимизирующую интегральные затраты с учетом дисконта. В частном случае, когда оборудование не подвержено старению, она формализована как задача оптимального управления с дополнительным условием возрастания функции. Получено аналитическое решение этой задачи методом дискретизации по времени и последующим решением методом динамического программирования. В общем случае динамическое программирование неприменимо, и дискретизация по времени позволяет получить лишь приближенное численное решение. Далее предполагается, что разные подотрасли имеют разные функции дисконта, описывающие зависимость полезности от динамики затрат с течением времени. В качестве принципа оптимальности используется компромиссное решение. В этом случае дискретизация по времени также позволяет получить приближенное численное решение.
\end{abstract}

Ключевые слова: макроэкономическая модель, оптимальное управление, замена оборудования, динамическая оптимизация, динамическое программирование, компромиссное решение.

\section{COMPROMISE SOLUTION IN AN ECONOMY MODEL WITH REPLACEMENT OF EQUIPMENT}

\author{
(c) 2019 O. A. MALAFEEV, A. P. PARFENOV
}

\begin{abstract}
In this paper, we formalize the problem on the optimal strategy for replacing equipment in a branch of economy in the presence of price expectations and expectations of Harrod-neutral technological progress. The problem is to determine a strategy for the purchase and write-off of equipment at each moment of time minimizing the integral costs taking into account the discount. In the particular case where the equipment is not subject to aging, the problem is formalized as an optimal control problem with an additional condition of increasing the function. An analytical solution of this problem is obtained by the time discretization method and subsequent solving by the dynamic programming method. In the general case, dynamic programming is not applicable, and time discretization provides only an approximate numerical solution. Further, we assume that various branches have different discount functions describing the dependence of utility on cost dynamics over time. As an optimality principle, we use a compromise solution. In this case, time discretization also allows one to obtain an approximate numerical solution.
\end{abstract}

Keywords and phrases: macroeconomic model, optimal control, replacement of equipment, dynamic optimization, dynamic programming, compromise solution.

AMS Subject Classification: 49L20, 91B38 
Введение. Динамические модели макроэкономики нередко формализуются как задачи оптимального управления. В более простых случаях предполагается, что все функции от времени задаются в определенной форме, а оптимизации подлежат лишь параметры модели. Так, в [3] формализована задача оптимальной стратегии замены оборудования в отрасли экономики при наличии ценовых ожиданий и ожиданий технического прогресса, нейтрального по Харроду. При этом предполагается, что выполнены следующие условия:

(1) отрасль производит однородные продукты, имеющие одинаковую ценность;

(2) оборудование, используемое в производстве, однородно, т.е., «машины» могут отличаться только сроком службы. Машина рассматривается как единое целое, т.е. она может быть отремонтирована или заменена только целиком, без учета деталей;

(3) отказ оборудования не влечет дополнительных потерь. Это значит, что профилактический ремонт не целесообразен: оборудование ремонтируется только в случае поломки;

(4) замена оборудования требует только затрат на покупку нового оборудования, которую можно производить, не останавливая производство;

(5) ценовые ожидания и ожидания технического прогресса описываются экспоненциальными функциями;

(6) количество продукции, выпускаемой в отрасли, задано (например, определяется спросом).

Полезность определяется динамикой прибыли, т.е. динамикой затрат при заданном количестве выпускаемой продукции. Определена интегральная полезность с дисконтом, причем функции дисконта для разных фирм в отрасли различны.

В [3] предполагается, что оборудование один раз куплено, а потом только обслуживается, и задача оптимизации сводится к определению количества купленного оборудования. В данной же работе рассмотрена динамика покупки и списания оборудования, а значит, и распределение оборудования по возрастам в каждый момент времени.

В разделе 1 формализована задача определения стратегии покупки и списания оборудования, минимизирующая интегральные затраты с учетом дисконта.

В разделе 2 рассмотрен частный случай, когда оборудование не подвержено старению. В этом случае получается задача оптимального управления с интегрально-терминальной целевой функцией (задача Больца) с дополнительным условием возрастания функции. Получено решение этой задачи методом дискретизации по времени и последующим решением методом динамического программирования. В этом случае получено аналитическое решение.

В разделе 3 рассмотрен общий случай. Показано, что динамическое программирование неприменимо, и дискретизация по времени позволяет получить лишь приближенное численное решение. Далее предполагается, что разные подотрасли имеют разные функции дисконта, описывающие зависимость полезности от динамики затрат с течением времени. В качестве принципа оптимальности используется компромиссное решение, согласующее интересы разных фирм. В этом случае дискретизация по времени также позволяет получить приближенное численное решение.

1. Описание общей модели. Пусть в отрасли производится один продукт в количестве

$$
X(t)=X_{0} e^{x t}
$$

в единицу времени, цена которого

$$
P(t)=P_{0} e^{p t} .
$$

Пусть $K(t, s)$ - это капитал, т.е. количество оборудования, купленного в момент времени $s$ и используемого в момент $t$, а $L(t, s)$ - это труд, т.е. количество рабочих, работающих в момент $t$ на оборудовании, купленном в момент $s$. Таким образом, функции $K, L$ неотрицательны и определены только при $t \geqslant s$. Пусть начальное распределение капитала по возрасту описывается функцией $K_{0}(s)$, где $s \leqslant 0$.

Пусть фиксированная доля $d$ оборудования в единицу времени выходит из строя, так что к моменту $t$ остается

$$
K(t, s)=e^{-d(t-s)}\left(K_{0}(s)+B(s)\right)-U(t, s),
$$


где $B(s)$ - количество оборудования, купленного в момент $s, U(t, s)$ - количество оборудования, купленного в момент $s$ и списанного к моменту $t$. Таким образом, функции $B(s)$ и $U(t, s)$ неотрицательны и определены при $s \geqslant 0, t \geqslant 0$. Функция $U(t, s)$ определена только при $t \geqslant s$, возрастает по $t$ и удовлетворяет неравенствам

$$
0 \leqslant U(t, s) \leqslant e^{-d(t-s)} B(s) .
$$

Тогда общее количество оборудования равно

$$
K(t)=\int_{0}^{t} K(t, s) d s=K_{0} e^{-d t}+\int_{0}^{t}\left(e^{-d(t-s)} B(s)-U(t, s)\right) d s,
$$

где

$$
K_{0}=\int_{0}^{t} e^{d s} K_{0}(s) d s .
$$

Общее количество рабочей силы равно

$$
L(t)=\int_{0}^{t} L(t, s) d s .
$$

Пусть производственная функция - это функция Кобба-Дугласа с учетом научно-технического прогресса (для нового оборудования), нейтрального по Харроду:

$$
X(K(t), L(t), t)=A K(t)^{a}\left(L(t) e^{g t}\right)^{1-a},
$$

т.е. прогресс связан с повышением эффективности рабочей силы.

Однако по мере старения оборудования нужно тратить все больше работы, чтобы получить тот же результат. Поэтому для более старого оборудования требуется больше рабочей силы. Пусть удельная норма труда на единицу капитала равна $L(t) / K(t)=l(t)$. Тогда без учета старения оборудования производственная функция (2) выглядела бы так:

$$
X(K(t), L(t), t)=A K(t)^{a}\left(l(t) K(t) e^{g t}\right)^{1-a}=A K(t) l(t)^{1-a} e^{(1-a) g t} .
$$

С учетом старения оборудования норма труда на единицу капитала для оборудования, купленного в момент $s$, умножается на $e^{m(t-s)}$. Следовательно, распределение рабочей силы определяется формулой

$$
L(t, s)=l(t) e^{m(t-s)} K(t, s) .
$$

Производственная функция с учетом старения оборудования имеет вид

$$
\begin{aligned}
& X(t)=X(K(t, s), L(t, s), t)=A K(t) l(t)^{1-a} e^{(1-a) g t}= \\
&=A l(t)^{1-a} e^{(1-a) g t}\left(K_{0} e^{-d t}+\int_{0}^{t}\left(e^{-d(t-s)} B(s)-U(t, s)\right) d s\right) .
\end{aligned}
$$

Стоимость оборудования и зарплата меняются по экспоненциальному закону:

$$
Q(t)=Q_{0} e^{q t}, \quad W(t)=W_{0} e^{w t},
$$

а процентная ставка на рынке равна $r$, т.е. стоимость денег меняется со временем как $e^{-r t}$.

Затраты на производство в единицу времени $C(t)$ зависят от функций $B, U, l$, а также от экзогенных параметров: 


$$
\begin{aligned}
C(t)=Q(t) & B(t)+L(t) W(t)=Q_{0} e^{q t} B(t)+W_{0} e^{w t} \int_{0}^{t} l(t) e^{m(t-s)} K(t, s) d s= \\
& =Q_{0} e^{q t} B(t)+W_{0} e^{(w+m) t} l(t) \int_{0}^{t} e^{-m s}\left(e^{-d t+d s}\left(K_{0}(s)+B(s)\right)-U(t, s)\right) d s= \\
& =Q_{0} e^{q t} B(t)+W_{0} e^{(w+m) t} l(t)\left(K_{0}^{*} e^{-d t}+e^{-d t} \int_{0}^{t} e^{(d-m) s} B(s) d s-\int_{0}^{t} e^{-m s} U(t, s) d s\right),
\end{aligned}
$$

где

$$
K_{0}^{*}=\int_{0}^{t} e^{(d-m) s} K_{0}(s) d s .
$$

\section{2. Модель без старения оборудования.}

2.1. Постановка задачи. Рассмотрим сперва частный случай, когда обслуживание оборудования не требует роста численности рабочей силы со временем, т.е. $m=0$. В этом случае не имеет смысла рассматривать распределение капитала и рабочей силы по возрастам $K(t, s)$ и $L(t, s)$ и начальное распределение капитала $K_{0}(s)$ : нам нужны только функции $K(t), L(t)$ и константа $K_{0}=K_{0}^{*}$. Производственная функция тогда описывается формулой (2).

Тогда для любого заданного $B(t)$ выгодно иметь $K(t)$ как можно больше, чтобы $L(t)$ было как можно меньше. Следовательно, в оптимальном случае оборудование не списывается: $U(t, s)=0$. В этом случае количество продукции описывается формулой

$$
\begin{aligned}
X(t)=A l(t)^{1-a} e^{(1-a) g t}\left(K_{0} e^{-d t}+\int_{0}^{t} e^{-d(t-s)} B(s) d s\right) & = \\
& =A l(t)^{1-a} e^{((1-a) g-d) t}\left(K_{0}+\int_{0}^{t} e^{d s} B(s) d s\right),
\end{aligned}
$$

откуда можно выразить $l(t)$ :

$$
l(t)=\frac{(X(t) / A)^{1 /(1-a)} e^{(-g+d /(1-a)) t}}{\left(K_{0}+\int_{0}^{t} e^{d s} B(s) d s\right)^{1 /(1-a)}}=\frac{X_{0}^{1 /(1-a)} A^{-1 /(1-a)} e^{(-g+(d+x) /(1-a)) t}}{\left(K_{0}+\int_{0}^{t} e^{d s} B(s) d s\right)^{1 /(1-a)}}
$$

Тогда затраты в момент $t$ равны

$$
\begin{aligned}
C(t)=Q_{0} e^{q t} B(t)+W_{0} e^{w t} l(t) & \int_{0}^{t} e^{-d t+d s} B(s) d s= \\
=Q_{0} e^{q t} B(t)+W_{0} e^{(w-d) t} l(t) \int_{0}^{t} e^{d s} B(s) d s= & \\
=Q_{0} e^{q t} B(t)+W_{0}^{*} e^{(w+(d a+x) /(1-a)-g) t} & \left(K_{0}+\int_{0}^{t} e^{d s} B(s) d s\right)^{-a /(1-a)}= \\
& =Q_{0} e^{q t} B(t)+\frac{W_{0}^{*} e^{(w+(d a+x) /(1-a)-g) t}}{\left(K_{0}+\int_{0}^{t} e^{d s} B(s) d s\right)^{a /(1-a)},}
\end{aligned}
$$


где

$$
W_{0}^{*}=W_{0} X_{0}^{1 /(1-a)} A^{-1 /(1-a)} .
$$

Осталось в каждый момент времени $t$ выбрать количество покупаемого оборудования $B(t)$, минимизирующее общие затраты с учетом процентной ставки:

$$
\int_{0}^{T} C(t) e^{-r t} d t
$$

Введем обозначение

$$
F(t)=\int_{0}^{t} e^{d s} B(s) d s
$$

Поскольку $B(s)$ - произвольная неотрицательная функция, $F(t)$ - произвольная неотрицательная возрастающая функция с условием $F(0)=0$. Тогда $B(t)=e^{-d t} F^{\prime}(t)$, и общие затраты равны

$$
\begin{gathered}
C=\int_{0}^{T}\left(Q_{0} e^{(q-d-r) t} F^{\prime}(t)+\frac{W_{0}^{*} e^{(w+(d a+x) /(1-a)-g-r) t}}{\left(K_{0}+F(t)\right)^{a /(1-a)}}\right) d t= \\
\quad=Q_{0} \int_{0}^{T} e^{(q-d-r) t} F^{\prime}(t) d t+W_{0}^{*} \int_{0}^{T} \frac{e^{(w+(d a+x) /(1-a)-g-r) t}}{\left(K_{0}+F(t)\right)^{a /(1-a)}} d t .
\end{gathered}
$$

Далее нам понадобится упрощение выражений вида $\int_{0}^{t} e^{c s} B(s) d s$. Упрощаем их интегрированием
по частям:

$$
\begin{array}{r}
\int_{0}^{t} e^{c s} B(s) d s=\int_{0}^{t} e^{(c-d) s} F^{\prime}(s) d s=\left(e^{(c-d) t} F(t)-e^{0} F(0)\right)-\int_{0}^{t}(c-d) e^{(c-d) s} F(s) d s= \\
=e^{(c-d) t} F(t)-(c-d) \int_{0}^{t} e^{(c-d) s} F(s) d s .
\end{array}
$$

Отсюда следует, что первое слагаемое равно

$$
Q_{0} \int_{0}^{T} e^{(q-r) t} B(t) d t=Q_{0} e^{(q-d-r) T} F(T)-Q_{0}(q-d-r) \int_{0}^{T} e^{(q-d-r) t} F(t) d t .
$$

Разделив целевую функцию на константу $Q_{0}$, получаем задачу Больца:

$$
\min _{F}\left(e^{(q-d-r) T} F(T)+\int_{0}^{T}\left((r+d-q) e^{(q-d-r) t} F(t)+\frac{W_{0}^{*}}{Q_{0}} \frac{e^{(w+(d a+x) /(1-a)-g-r) t}}{\left(K_{0}+F(t)\right)^{a /(1-a)}}\right) d t\right)
$$

для возрастающей функции $F$ с начальным условием $F(0)=0$.

Если $r+d-q>0$, то в каждый момент времени $t$ подынтегральная функция

$$
f(F(t), t)=(r+d-q) e^{(q-d-r) t} F(t)+\frac{W_{0}^{*}}{Q_{0}} \frac{e^{(w+(d a+x) /(1-a)-g-r) t}}{\left(K_{0}+F(t)\right)^{a /(1-a)}}
$$

достигает минимума по $F(t)$ при

$$
F(t)=\left(\frac{a W_{0}^{*}}{(1-a) Q_{0}(r+d-q)}\right)^{1-a} e^{((w-q-g)(1-a)+d+x) t}-K_{0}
$$


Эта функция была бы решением задачи, если бы не условие возрастания $F(t)$ и не слагаемое $e^{(q-d-r) T} F(T)$, которое также нужно минимизировать. Поэтому в общем случае решение задачи нетривиально, и его вид существенно зависит от параметров $q, d, r, w, a, g, x$.

Если же $r+d-q<0$, то подынтегральная функция убывает, а значит, ее минимум достигается при $F(t)=0$. Этот случай рассмотрен в следующем разделе.

2.2. Случаи, когда решением является константа. Рассмотрим два случая, когда решением задачи (6) является постоянная функция $F(t)=$ const: оборудование вообще невыгодно покупать либо выгодно купить все сразу, а потом не покупать.

1. Пусть для всех $t \leqslant T$ выполняется неравенство

$$
\left(\frac{a /(1-a) W_{0}^{*}}{Q_{0}(r+d-q)}\right)^{1-a} e^{((w-q-g)(1-a)+d+x) t} \leqslant K_{0},
$$

т.е. в каждый момент времени $t$ выгодно иметь меньше оборудования, чем реально имеется. Тогда решением задачи является постоянная функция $F(t)=0$, т.е. невыгодно закупать дополнительное оборудование.

2. Пусть $r+d-q \leqslant 0$, т.е. реальные цены на оборудование растут быстрее, чем оно выходит из строя. Тогда подынтегральная функция (7) для всех $t$ убывает по $F(t)$. При этом $F(t)$ ограничена сверху только при условиями возрастания функции $F$. Следовательно, минимум достигается при $F(t)=$ const $=F(T)$.

Иначе говоря, выгодно закупить все оборудование сразу, в момент времени 0. Математически это означает, что закупки $B(t)$ - импульсная функция в точке 0 , а во всех остальных точках она равна 0.

3. Пусть $d=0,(w-q-g)(1-a)+x=0$, т.е. в каждый момент времени выгодно иметь одно и то же количество оборудования, и

$$
\left(\frac{a /(1-a) W_{0}^{*}}{Q_{0}(r-q)}\right)^{1-a}>K_{0}
$$

т.е. это количество больше, чем имелось в начале. Тогда при отсутствии постоянного слагаемого $e^{(q-d-r) T} F(T)$ минимум достигается при постоянной функции

$$
F(t)=\left(\frac{a /(1-a) W_{0}^{*}}{Q_{0}(r-q)}\right)^{1-a}-K_{0}=F_{0},
$$

поскольку это значение минимизирует подынтегральную функцию при любом $t$.

Добавление слагаемого $e^{(q-d-r) T} F(T)$, вообще говоря, уменьшает все значения $F(t)$, причем они уменьшаются все одновременно, поскольку при уменьшении $F(T)$ до значения, меньшего, чем $F_{0}$, имеет смысл все остальные $F(t)$ оставить как можно ближе к этом значению, минимизирующему значение подынтегральной функции при любом $t$, а значит, все они в оптимальном случае все равно равны $F(T)$.

Как выглядит решение задачи (6) при $F(t)=F(T)=F$ ? Значение константы $F$ должно минимизировать функцию

$$
\begin{gathered}
f(F)=e^{(q-d-r) T} F+\int_{0}^{T}\left((r+d-q) e^{(q-d-r) t} F+\frac{W_{0}^{*}}{Q_{0}} \frac{e^{(w+(d a+x) /(1-a)-g-r) t}}{\left(K_{0}+F\right)^{a /(1-a)}}\right) d t= \\
=\left(e^{(q-d-r) T}-(q-d-r) \int_{0}^{T} e^{(q-d-r) t} d t\right) F+\frac{W_{0}^{*}}{Q_{0}\left(K_{0}+F\right)^{a /(1-a)}} \int_{0}^{T} e^{(w+(d a+x) /(1-a)-g-r) t} d t= \\
=F+\frac{e^{m T}-1}{m} \frac{W_{0}^{*}}{Q_{0}\left(K_{0}+F\right)^{a /(1-a)}},
\end{gathered}
$$

где $m=w+(d a+x) /(1-a)-g-r$. Если $m=0$, то вместо $\left(e^{m T}-1\right) / m$ нужно подставить $T$. Но при любом $m$ выполняется неравенство $\left(e^{m T}-1\right) / m>0$. 
Функция $f(F)$ выпукла и ее минимум достигается при таком $F^{*}$, что $f^{\prime}\left(F^{*}\right)=0$, т.е.

$$
1-\frac{e^{m T}-1}{m} \frac{a(1-a) W_{0}^{*}}{Q_{0}\left(K_{0}+F^{*}\right)^{1 /(1-a)}}=0 \quad \Longleftrightarrow \quad F^{*}=\left(\frac{e^{m T}-1}{m} \frac{a(1-a) W_{0}^{*}}{Q_{0}}\right)^{1-a}-K_{0} .
$$

Возможны два варианта:

(1) $F^{*} \leqslant 0$; тогда закупка оборудования нецелесообразна, а значит, $B(s)=0$;

(2) $F^{*}>0$; тогда закупка оборудования выполняется только в начальный момент времени, $B(s)=F^{*} \delta(s)$.

2.3. Дискретизачия задачи по времени. Далее предполагаем, что $r+d-q>0$, поскольку, как мы уже выяснили, в противном случае решением является закупка всего оборудования в начальный момент времени. Если же $r+d-q>0$, то решение задачи не обязательно постоянно.

Пример. Пусть значения функции (8) возрастают по $t$; это условие выполняется, например, при

$$
\left(\frac{a /(1-a) W_{0}^{*}}{Q_{0}(r+d-q)}\right)^{1-a} \geqslant K_{0}
$$

и $(w-q-g)(1-a)+x \geqslant 0$. Тогда при отсутствии слагаемого $e^{(q-d-r) T} F(T)$ оптимальным значением $F(t)$ в каждой точке при переходе к пределу будет возрастающая функция (8); в данном случае, добавление условий возрастания $F(t)$ не мешает ему доставлять минимум подынтегральной функции при любом $t$. Добавление слагаемого $e^{(q-d-r) T} F(T)$ приводит к тому, что оптимальная функция оказывается меньше.

Далее нам понадобится ограничение на $F(t)$ сверху. Поскольку $F$ возрастает, достаточно получить ограничение сверху для $F(T)$. Для этого можно взять его максимально возможное оптимальное значение, которое, согласно формуле (8), равно

$$
M=\left(\frac{a /(1-a) W_{0}^{*}}{Q_{0}(r+d-q)}\right)^{1-a} \max \left\{e^{((w-q-g)(1-a)+d+x) T}, 1\right\}-K_{0} .
$$

Дополнительные условия возрастания $F(t)$ и дополнительное слагаемое $F(T)$ могут лишь уменьшить оптимальные значения $F(t)$, но никак не увеличить.

Также нам понадобятся ограничения сверху на производную подынтегральной функции $f$ по первому и второму аргументу:

$$
\begin{aligned}
& L_{1} \leqslant \max _{t \in[0 ; T]} \mid(r+d-q) e^{(q-d-r) t}-\frac{a W_{0}^{*}}{(1-a) Q_{0}} \frac{e^{(w+(d a+x) /(1-a)-g-r) t}}{\left(K_{0}+F(t)\right)^{1 /(1-a)}} \mid \leqslant \\
& \leqslant \max _{t \in[0 ; T]}\left((r+d-q) e^{(q-d-r) t}+\frac{a W_{0}^{*}}{(1-a) Q_{0}} \frac{e^{(w+(d a+x) /(1-a)-g-r) t}}{\left(K_{0}+F(t)\right)^{1 /(1-a)}}\right) \leqslant \\
& \leqslant(r+d-q)+\frac{a W_{0}^{*}}{(1-a) Q_{0}} \frac{\max \left\{e^{(w+(d a+x) /(1-a)-g-r) T, 1}\right\}}{K_{0}^{1 /(1-a)}}
\end{aligned}
$$

и

$$
\begin{gathered}
L_{2} \leqslant \max _{t \in[0 ; T]}\left|(r+d-q)^{2} e^{(q-d-r) t} F(t)-\frac{W_{0}^{*}}{Q_{0}} \frac{\left(w+\frac{d a+x}{1-a}-g-r\right) e^{(w+(d a+x) /(1-a)-g-r) t}}{\left(K_{0}+F(t)\right)^{a /(1-a)}}\right| \leqslant \\
\leqslant \max _{t \in[0 ; T]}\left((r+d-q)^{2} e^{(q-d-r) t} F(t)+\frac{W_{0}^{*}\left(w+\frac{d a+x}{1-a}-g-r\right)}{Q_{0}} \frac{e^{(w+(d a+x) /(1-a)-g-r) t}}{\left(K_{0}+F(t)\right)^{a /(1-a)}}\right) \leqslant \\
\leqslant(r+d-q)^{2} F(T)+\frac{W_{0}^{*}\left(w+\frac{d a+x}{1-a}-g-r\right) \max \left\{e^{(w+(d a+x) /(1-a)-g-r) T}, 1\right\}}{Q_{0} K_{0}^{a /(1-a)}} .
\end{gathered}
$$


Здесь при ограничении сверху мы заменили $F(t)$ на большее значение $F(T)$ в числителе и на меньшее значение 0 в знаменателе. Далее, подставив вместо $F(T)$ его верхнюю границу $M$, получим

$$
\begin{aligned}
L_{1} \leqslant(r+d-q) & +\frac{a W_{0}^{*}}{(1-a) Q_{0}} \frac{\max \left\{e^{(w+(d a+x) /(1-a)-g-r) T}, 1\right\}}{K_{0}^{1 /(1-a)}}, \\
L_{2} \leqslant(r+d-q)^{2} & \left(\left(\frac{a /(1-a) W_{0}^{*}}{Q_{0}(r+d-q)}\right)^{1-a} \max \left\{e^{((w-q-g)(1-a)+d+x) T}, 1\right\}-K_{0}\right)+ \\
& +\frac{W_{0}^{*}\left(w+\frac{d a+x}{1-a}-g-r\right) \max \left\{e^{(w+(d a+x) /(1-a)-g-r) T}, 1\right\}}{Q_{0} K_{0}^{a /(1-a)}} .
\end{aligned}
$$

Пусть время дискретно, т.е. все изменения могут происходить только в моменты времени, кратные $\delta=T / n$, т.е. в моменты $0, \delta, \ldots, \delta k, \ldots, \delta n=T$. Тогда исходную задачу минимизации можно приблизить задачей с дискретным временем

$$
\min _{x_{1}, \ldots, x_{n}}\left(x_{n}+\sum_{i=1}^{n-1} a_{k} x_{k}+\frac{b_{k}}{\left(x_{k}+K_{0}\right)^{a /(1-a)}}\right)
$$

при условиях $x_{i+1} \geqslant x_{i}$ и заданном $x_{0}=0$, где

$$
a_{k}=\frac{(r+d-q) e^{(q-d-r) \delta k}}{e^{(q-d-r) T}}, \quad b_{k}=\frac{W_{0}^{*} e^{(w+(d a+x) /(1-a)-g-r) \delta k}}{Q_{0} e^{(q-d-r) T}} .
$$

Замена непрерывной функции $F(t)$ кусочно-постоянной функцией $x(t)$ приводит к погрешности в определении целевой функции. Эта погрешность определяется только погрешностью приближения интеграла суммой (поскольку равенство $x_{n}=F(T)$ выполняется точно). Подынтегральная функция $f(F(t), t)$, заданная формулой $(7)$, при $t \in[0 ; T]$ удовлетворяет условию Липшица с константами $L_{1}, L_{2}$, в следующем смысле:

$$
\left|f\left(x+h_{1}, t+h_{2}\right)-f(x, t)\right| \leqslant L_{1}\left|h_{1}\right|+L_{2}\left|h_{2}\right|,
$$

где в качестве $L_{1}, L_{2}$ можно взять максимум по $[0 ; T]$ модулей ее частных производных по $F(t)$ и по $t$, определяемые по формулам (10). Тогда погрешность замены интеграла суммой не превосходит величины

$$
\begin{gathered}
\left|\int_{0}^{T} f(F(t), t) d t-\delta \sum_{k=0}^{n-1} f\left(x_{k}, \delta k\right)\right| \leqslant \\
\leqslant \sum_{k=0}^{n-1} \int_{\delta k}^{\delta(k+1)}\left|f(F(t), t)-f\left(x_{k}, \delta k\right)\right| d t \leqslant \sum_{k=0}^{n-1} \int_{\delta k}^{\delta(k+1)}\left(L_{1}\left|F(t)-x_{k}\right|+L_{2}|t-\delta k|\right) d t \leqslant \\
\leqslant L_{1} \sum_{k=0}^{n-1} \int_{\delta k}^{\delta(k+1)}\left(x_{k+1}-x_{k}\right) d t+L_{2} \sum_{k=0}^{n-1} \int_{\delta k}^{\delta(k+1)}(t-\delta k) d t= \\
=L_{1} \delta F(T)+\frac{1}{2} L_{2} \delta T \leqslant \delta\left(L_{1} M+\frac{1}{2} L_{2} T\right)=\frac{T}{n}\left(L_{1} M+\frac{1}{2} L_{2} T\right) \underset{n \rightarrow \infty}{\longrightarrow} 0 .
\end{gathered}
$$

Заметим, что погрешность в определении целевой функции стремится к 0, даже несмотря на то, что $F(t)$ может возрастать скачкообразно, т.е. возможны импульсные управления. 
2.4. Решение методом динамического программирования. Задачу с дискретным временем можно приближенно решить методом динамического программирования (см. [1]). Обозначим через $d_{n}(c)$ решение исходной задачи минимизации с $n$ слагаемыми при $x_{0}=c$, а через $d_{k}(c)-$ решение той же задачи, в которой взяты последние $k$ слагаемых, при $x_{n-k}=c$. Очевидно, функция $d_{k}$ удовлетворяет рекуррентному соотношению

$$
d_{k}(c)=\min _{x \geqslant c}\left(a_{n-k+1} x+\frac{b_{n-k+1}}{\left(x+K_{0}\right)^{a /(1-a)}}+d_{k-1}(x)\right)
$$

с начальным условием

$$
d_{1}(c)=\min _{x \geqslant c} \frac{x}{\delta}=\frac{c}{\delta} .
$$

Предположив дифференцируемость $F$ и переходя к пределу при $\delta \rightarrow 0$, из условий (11) можно получить уравнение Беллмана (см. [1]). Однако решать уравнение в частных производных достаточно сложно, тем более, что функция $F$ может быть разрывной. Поэтому найдем решение уравнения с дискретным временем, после чего перейдем к пределу.

Найдем значение функции $d_{2}(c)$ :

$$
d_{2}(c)=\min _{x \geqslant c}\left(a_{n-1} x+\frac{b_{n-1}}{\left(x+K_{0}\right)^{a /(1-a)}}+\frac{x}{\delta}\right)=\min _{x \geqslant c}\left(\left(a_{n-1}+\frac{1}{\delta}\right) x+\frac{b_{n-1}}{\left(x+K_{0}\right)^{a /(1-a)}}\right) .
$$

Очевидно, оно зависит от того, больше или меньше $c$ точки локального минимума

$$
c_{1}^{*}=\left(\frac{a /(1-a) b_{n-1}}{a_{n-1}+1 / \delta}\right)^{1-a}-K_{0} .
$$

Следовательно, значение функции $d_{2}(c)$ равно

$$
d_{2}(c)= \begin{cases}\frac{b_{n-1}^{1-a}\left(a_{n-1}+1 / \delta\right)^{a}}{a^{a}(1-a)^{1-a}}-\left(a_{n-1}+\frac{1}{\delta}\right) K_{0}, & c<{ }_{1}^{*}, \\ \left(a_{n-1}+\frac{1}{\delta}\right) c+\frac{b_{n-1}}{\left(c+K_{0}\right)^{a /(1-a)}}, & c \geqslant c_{1}^{*} .\end{cases}
$$

Это возрастающая выпуклая функция, которая до точки «склеивания» $c_{1}^{*}$ равна константе.

При достаточно малом $\delta$ значения $a_{n-2}$ и $a_{n-1}, b_{n-2}$ и $b_{n-1}$ различаются незначительно, а значит,

$$
\left(\frac{a b_{n-2}}{(1-a) a_{n-2}}\right)^{1-a}-K_{0}>\left(\frac{a b_{n-1}}{(1-a)\left(a_{n-1}+1 / \delta\right)}\right)^{1-a}-K_{0},
$$

т.е. минимум функции

$$
a_{n-2} x+\frac{b_{n-2}}{\left(x+K_{0}\right)^{a /(1-a)}}
$$

достигается при большем значении $x$, чем точка «склеивания» функции $d_{2}(c)$. Следовательно, минимум функции $d_{3}(c)$ достигается на участке между этими двумя точками, где целевая функция имеет вид

$$
\left(a_{n-2}+a_{n-1}+\frac{1}{\delta}\right) x+\frac{b_{n-1}+b_{n-2}}{\left(x+K_{0}\right)^{a /(1-a)}},
$$

и равен он

$$
c_{2}^{*}=\left(\frac{a /(1-a)\left(b_{n-2}+b_{n-1}\right)}{a_{n-2}+a_{n-1}+\frac{1}{\delta}}\right)^{1-a}-K_{0} .
$$

Утверждение 1. Функиия $d_{k}(c)$ обладает следующими свойствами:

(1) $d_{k}($ c) возрастает;

(2) $d_{k}(c)$ выпукла; 
(3) существуют такие константа $C^{*}$, перестановка $\left(i_{1}, \ldots, i_{k-1}\right)$ и последовательность точек $c_{i_{1}}^{*} \leqslant \ldots \leqslant c_{i_{k-1}}^{*}$, что функиия $d_{k}(c)$ имеет вид

$$
d_{k}(c)=\left\{\left(\sum_{j: c>c_{i_{j}}} a_{n-i_{j}}+\frac{1}{\delta}\right) c+\frac{1}{\left(c+K_{0}\right)^{a /(1-a)}} \sum_{j: c>c_{i_{j}}} b_{n-i_{j}}, \quad c \geqslant c_{i_{1}}^{*} .\right.
$$

Доказательство. (1) Функция $d_{k}(c)$ возрастает, поскольку это минимум по множеству $x \geqslant c$, которое уменьшается при росте $c$.

(2) Применим индукцию по $k$. База индукции: функция $d_{1}(c)$ линейна, а значит, выпукла. Предположим, что функция $d_{k-1}$ выпукла. Тогда функция $d_{k}$ выпукла как минимум выпуклой функции

$$
a_{n-k+1} x+\frac{b_{n-k+1}}{\left(x+c_{n-k+1}\right)^{a /(1-a)}}+d_{k-1}(x)
$$

по многогранному множеству, заданному линейным неравенством $x \geqslant c$.

(3) Также применим индукцию. База индукции: для функции $d_{2}(c)$, как мы выяснили, существуют такие константа

$$
C^{*}=\frac{b_{n-1}^{1-a}\left(a_{n-1}+1 / \delta\right)^{a}}{a^{a}(1-a)^{1-a}}-\left(a_{n-1}+\frac{1}{\delta}\right) K_{0}
$$

и точка $c_{1}^{*}$, что функция $d_{2}(c)$ имеет вид $(12)$. Предположим, что такие константа и последовательность точек существуют для функции $d_{k-1}(c)$, и докажем, что они существуют для функции $d_{k}(c)$.

Функция $d_{k}(c)$ задается рекуррентным соотношением (11). Найдем ее минимум для $c=0$. Поскольку минимум ищется по выпуклой функции, он достигается в единственной точке локального минимума, совпадающей с точкой глобального минимума. Чтобы его найти, достаточно перебрать все отрезки $\left[c_{i_{j}}^{*} ; c_{i_{j+1}}^{*}\right]$, полагая $c_{i_{0}}^{*}=0$. За достаточный же для всех $k$ правый край (т.е. максимально возможное значение $F(t)$ для всех возможных $t$ ) можно взять максимально возможное значение $M$ оптимального $F(T)$, которое, определяется по формуле (9). На каждом отрезке найдем минимум выражения (11) и сравним эти минимумы. Получившийся глобальный минимум достигается в точке $c_{k-1}^{*}$, которая может совпадать с одной из точек $c_{i_{j}}^{*}$, а может не совпадать ни с одной из них. После этого всем точкам $c_{i_{j}}^{*}$, которые меньше $c_{k-1}^{*}$, присваиваем новое значение $c_{k-1}^{*}$, а значения остальных точек $c_{i_{j}}^{*}$ оставляем неизменными.

Теперь, очевидно, для всех $c \leqslant c_{k-1}^{*}$ функция $d_{k}(c)$ равна константе, а именно, минимальному значению (11). Если же $c>c_{k-1}^{*}$, то минимизируемая функция после точки глобального минимума возрастает, а значит, ее минимум по $x \geqslant c$ достигается в левом крае, т.е. в точке $c$. Это значит, что $d_{k}(c)$ на каждом отрезке $\left[c_{i_{j}}^{*} ; c_{i_{j+1}}^{*}\right]$ равно сумме $d_{k-1}(c)$ и выражения

$$
a_{n-k+1} x+\frac{b_{n-k+1}}{\left(x+K_{0}\right)^{a /(1-a)}},
$$

откуда и получаем формулу (12).

Доказательство п. (3) утверждения дает алгоритм нахождения функций $d_{k}(c)$ и, в конечном счете, функции $d_{n}(c)$, дающей приближенное решение исходной задачи. Для этого нужно на $k$-м шаге решить $O(k)$ задач нахождения минимума, легко решаемых аналитически. Значит, общее число шагов алгоритма имеет порядок $O\left(n^{2}\right)$.

При этом из выражения для функции $d_{3}$ мы видим, что выражения для нескольких последующих функций будут аналогичными, и определенное количество начальных шагов, пока выполняется неравенство

$$
\frac{b_{n-k}}{a_{n-k}}>\frac{b_{n-k+1}+\cdots+b_{n-1}}{a_{n-k+1}+\cdots+a_{n-1}+1 / \delta}
$$


значение точки минимума $c_{k-1}^{*}$ будет возрастать по $k$, а все предыдущие точки $c_{j}^{*}$ будут «склеиваться» с этой точкой минимума, так что функция $d_{k}(c)$ каждый раз состоит всего из двух кусков. При этом, если последовательность $b_{k}$ в числителе убывает не медленнее, чем последовательность $a_{k}$ в знаменателе, т.е. если $w+(d+x) /(1-a) \leqslant q+g$, то последовательность сумм $b_{k}$ с убывающими номерами растет не медленнее, чем последовательность сумм $a_{k}$ с убывающими номерами, а значит, неравенство (13) будет выполняться вплоть до последней итерации. В этом случае все значения $c_{k}^{*}$ равны друг другу, а значит, переходя к пределу, получаем, что $F(t)$ равно константе.

Но если последовательность $b_{k}$ растет достаточно быстро, то начиная с определенного $k$, значения $c_{k-1}^{*}$ начнут убывать согласно формуле

$$
c_{k}^{*}=\left(\frac{a b_{n-k}}{(1-a) a_{n-k}}\right)^{1-a}-K_{0} .
$$

В этом случае $F(t)$ уже не равно константе, т.е. до определенного момента времени оказывается выгодна закупка нового оборудования. Переходя к пределу при $n \rightarrow \infty$, получаем, что $F(t)$ до определенного момента $t^{*}$ определяется по формуле (8), и только при $t>t^{*}$ равна константе. Момент $t^{*}$ можно приближенно определить численно по указанному выше алгоритму, но его можно найти и аналитически. Действительно, перейдя к пределу при $n \rightarrow \infty$, получим, что для $t^{*}$ должно выполняться неравенство

$$
\begin{aligned}
& \frac{W_{0}^{*} e^{(w+(d a+x) /(1-a)-g-r) t^{*}}}{Q_{0}(r+d-q) e^{(q-d-r) t^{*}}}>\frac{\int_{t^{*}}^{T} W_{0}^{*} e^{(w+(d a+x) /(1-a)-g-r) t} d t}{Q_{0}\left(e^{(q-d-r) T}+\int_{t^{*}}^{T}(r+d-q) e^{(q-d-r) t} d t\right)} \Longleftrightarrow \\
& \Longleftrightarrow \quad \frac{e^{(w+(d+x) /(1-a)-g-q) t^{*}}}{(r+d-q)}>\frac{e^{(w+(d a+x) /(1-a)-g-r) T}-e^{(w+(d a+x) /(1-a)-g-r) t^{*}}}{\left(w+\frac{d a+x}{1-a}-g-r\right)\left(e^{(q-d-r) T}-e^{(q-d-r) T}+e^{(q-d-r) t^{*}}\right)} \quad \Longleftrightarrow \\
& \Longleftrightarrow \quad \frac{e^{(w+(d a+x) /(1-a)-g-r) t^{*}}}{(r+d-q)}>\frac{e^{(w+(d a+x) /(1-a)-g-r) T}-e^{(w+(d a+x) /(1-a)-g-r) t^{*}}}{\left(w+\frac{d a+x}{1-a}-g-r\right)} .
\end{aligned}
$$

Введя обозначения

$$
r+d-q=u>0, \quad w+\frac{d a+x}{1-a}-g-r=z, \quad e^{(w+(d a+x) /(1-a)-g-r) t^{*}}=e^{z t^{*}}=z^{*}
$$

и домножив обе части неравенства на положительное число $u$, получим неравенство

$$
y^{*}>\frac{u}{z}\left(e^{z T}-y^{*}\right) \quad \Longleftrightarrow \quad y^{*}\left(1+\frac{u}{z}\right)>\frac{u}{z} e^{z T} .
$$

Возможны 3 варианта:

Вариант 1: $z>0$, следовательно, $1+u / z>0$; поделив обе части неравенства на $1+u / z=$ $(z+u) / z$, получаем

$$
y^{*}>\frac{u}{z+u} e^{z T} \Longleftrightarrow z t^{*}>z T-\ln \frac{z+u}{u} \Longleftrightarrow t^{*}>T-\frac{1}{z} \ln \frac{z+u}{u} .
$$

Если $\frac{1}{z} \ln \frac{z+u}{u}<T$, то до момента $T-\frac{1}{z} \ln \frac{z+u}{u}$ функция $F(t)$ растет согласно формуле (8), а после него равна константе.

Вариант 2: $z<0$, но $1+u / z>0$; тогда $z+u<0$, и неравенство

$$
y^{*}>\frac{u}{z+u} e^{z T}
$$

выполняется при любом $y^{*}>0$, поскольку в правой части неравенства стоит отрицательное число. Следовательно, при всех $t$ функция $F(t)$ постоянна. 
Вариант 3: $z<0$ и $1+u / z<0$; тогда $z+u>0$ и

$$
y^{*}<\frac{u}{z+u} e^{z T} \Longleftrightarrow z t^{*}<z T+\ln \frac{u}{z+u} \Longleftrightarrow t^{*}>T-\frac{1}{-z} \ln \frac{u}{z+u} .
$$

Если $\frac{1}{-z} \ln \frac{u}{z+u}<T$, то до момента $T-\frac{1}{-z} \ln \frac{u}{z+u}$ функция $F(t)$ растет согласно формуле $(8)$, а после него равна константе.

\section{3. Модель со старением, но без утилизации.}

3.1. Модель с износом оборудования. Рассмотрим теперь общую модель, в которой $m>0$ : со временем оборудование изнашивается, а значит, оборудование разного возраста различается. В этом случае нужно найти функции $B(s)$ и $U(s, t)$, минимизирующие интегральные затраты

$$
\begin{aligned}
C=\int_{0}^{T} C(t) e^{-r t} d t & =Q_{0} \int_{0}^{T} e^{(q-r) t} B(t) d t+ \\
& +W_{0} \int_{0}^{T} e^{(w+m-r) t} l(t)\left(K_{0}^{*} e^{-d t}+e^{-d t} \int_{0}^{t} e^{(d-m) s} B(s) d s-\int_{0}^{t} e^{-m s} U(t, s) d s\right) d t
\end{aligned}
$$

при условии

$$
\begin{gathered}
X_{0} e^{x t}=A l(t)^{1-a} e^{(1-a) g t}\left(K_{0} e^{-d t}+\int_{0}^{t}\left(e^{-d(t-s)} B(s)-U(t, s)\right) d s\right)= \\
=A l(t)^{1-a} e^{(1-a) g t}\left(K_{0} e^{-d t}+e^{-d t} \int_{0}^{t} e^{d s} B(s) d s-\int_{0}^{t} U(t, s) d s\right) .
\end{gathered}
$$

Отсюда можем выразить $l(t)$ :

$$
\begin{gathered}
l(t)=\left(\frac{1}{A} \frac{X_{0} e^{(x-(1-a) g) t}}{K_{0} e^{-d t}+e^{-d t} \int_{0}^{t} e^{d s} B(s) d s-\int_{0}^{t} U(t, s) d s}\right)^{1 /(1-a)}= \\
=A^{-1 /(1-a)} X_{0}^{1 /(1-a)} e^{(x /(1-a)-g) t}\left(K_{0} e^{-d t}+e^{-d t} \int_{0}^{t} e^{d s} B(s) d s-\int_{0}^{t} U(t, s) d s\right)^{-1 /(1-a)} .
\end{gathered}
$$

Подставляем $l(t)$ в выражение для общих затрат $C$ и получаем

$$
\begin{aligned}
& C=Q_{0} \int_{0}^{T} e^{(q-r) t} B(t) d t+ \\
& \quad+W_{0}^{*} \int_{0}^{T} e^{(w+m-r+x /(1-a)-g) t} \frac{K_{0}^{*} e^{-d t}+e^{-d t} \int_{0}^{t} e^{(d-m) s} B(s) d s-\int_{0}^{t} e^{-m s} U(t, s) d s}{\left(K_{0} e^{-d t}+e^{-d t} \int_{0}^{t} e^{d s} B(s) d s-\int_{0}^{t} U(t, s) d s\right)^{1 /(1-a)}} d t .
\end{aligned}
$$

Далее рассмотрим упрощенную модель, в которой оборудование нельзя утилизировать, т.е. $U(t, s)=0$. В этом случае выражение для затрат (14) упрощается: 


$$
\begin{gathered}
C=Q_{0} \int_{0}^{T} e^{(q-r) t} B(t) d t+W_{0}^{*} \int_{0}^{T} e^{(w+m-r+x /(1-a)-g) t} \frac{K_{0}^{*} e^{-d t}+e^{-d t} \int_{0}^{t} e^{(d-m) s} B(s) d s}{\left(K_{0} e^{-d t}+e^{-d t} \int_{0}^{t} e^{d s} B(s) d s\right)^{1 /(1-a)}} d t= \\
=Q_{0} \int_{0}^{T} e^{(q-r) t} B(t) d t+W_{0}^{*} \int_{0}^{T} e^{(w+m-r+(x+d a) /(1-a)-g) t} \frac{K_{0}^{*}+\int_{0}^{t} e^{(d-m) s} B(s) d s}{\left(K_{0}+\int_{0}^{t} e^{d s} B(s) d s\right)^{1 /(1-a)}} d t .
\end{gathered}
$$

Аналогично модели без старения оборудования, введем функцию $F(t)$ по формуле (4) и упростим соответствующие интегралы согласно формуле (5). Получим следующее выражение для затрат:

$$
\begin{aligned}
C=Q_{0} e^{(q-d-r) T} F(T)- & Q_{0}(q-d-r) \int_{0}^{T} e^{(q-d-r) t} F(t) d t+ \\
& +W_{0}^{*} \int_{0}^{T} e^{(w+m-r+(x+d a) /(1-a)-g) t} \frac{K_{0}^{*}+e^{-m t} F(t)+m \int_{0}^{t} e^{-m s} F(s) d s}{\left(K_{0}+F(t)\right)^{1 /(1-a)}} d t .
\end{aligned}
$$

Разделив целевую функцию на $Q_{0}$, получим задачу, аналогичную задаче Больца, но с дополнительным слагаемым, содержащим вложенный интеграл:

$$
\begin{aligned}
C=e^{(q-d-r) T} F(T)+ & \int_{0}^{T}\left((r+d-q) e^{(q-d-r) t} F(t)+\right. \\
& +\frac{W_{0}^{*}}{Q_{0}} e^{(w+m-r+(x+d a) /(1-a)-g) t} \frac{K_{0}^{*}+e^{-m t} F(t)}{\left.\left(K_{0}+F(t)\right)^{1 /(1-a)}\right) d t+} \\
& +\frac{W_{0}^{*} m}{Q_{0}} \int_{0}^{T} \frac{e^{(w+m-r+(x+d a) /(1-a)-g) t} \int_{0}^{t} e^{-m s} F(s) d s}{\left(K_{0}+F(t)\right)^{1 /(1-a)}} d t .
\end{aligned}
$$

3.2. Решение задачи методом дискретизачии. Задачу (15) также можно решить методом дискретизации по времени. Разбив отрезок $[0 ; T]$ на $n$ отрезков величины $\delta$, получим дискретную задачу минимизации по $x_{1}, \ldots, x_{n}$ :

$$
\begin{aligned}
& \min _{0<\leqslant x_{1} \leqslant \ldots \leqslant x_{n}}\left\{e^{(q-d-r) T} x_{n}+\delta \sum_{k=1}^{n}\left((r+d-q) e^{(q-d-r) \delta k} x_{k}+\right.\right. \\
& \left.+\frac{W_{0}^{*}}{Q_{0}} e^{(w+m-r+(x+d a) /(1-a)-g) \delta k} \frac{K_{0}^{*}+e^{-m \delta k} x_{k}}{\left(K_{0}+x_{k}\right)^{1 /(1-a)}}\right)+ \\
& \left.+\frac{W_{0}^{*} m}{Q_{0}} \delta^{2} \sum_{k=1}^{n} \frac{e^{(w+m-r+(x+d a) /(1-a)-g) \delta k} \sum_{j=1}^{k} e^{-m \delta j} x_{j}}{\left(K_{0}+x_{k}\right)^{1 /(1-a)}}\right\} .
\end{aligned}
$$

В данном случае каждое слагаемое, зависящее от $x_{k}$, зависит еще и от всех $x_{i}$ при $i<k$, а значит, задачу нельзя решить методом динамического программирования.

Это $n$-мерная задача минимизации выпуклой функции при линейных ограничениях. Ее можно решить методом условного градиента. Начальное решение: не покупать новое оборудование. Затем на каждом шаге нужно решить $n$-мерную задачу линейного программирования, определяя направление движения, и в данном направлении приближенно решить задачу минимизации одномерной выпуклой функции методом деления пополам. 
3.3. Разная интегральная полезность в разных подотраслях. Пусть отрасль делится на $s$ подотраслей или фирм. В каждой из них выпуск $X_{1}(t), \ldots, X_{s}(t)$ и затраты $C_{1}(t), \ldots, C_{s}(t)$ пропорциональны общим затратам с постоянным коэффициентом пропорциональности:

$$
C_{k}(t)=c_{k} C(t), \quad X_{k}(t)=x_{k} X(t) .
$$

Следовательно прибыль $k$-й подотрасли однозначно определяется ее затратами. При этом полезность от прибыли, равно как и полезность с отрицательным знаком от затрат, у $k$-й подотрасли является функционалом от функции дисконта, определенной от 0 до момента $T$ :

$$
C_{k}=\int_{0}^{T} C_{k}(t) f_{k}(t) d t=c_{k} \int_{0}^{T} C_{k}(t) f_{k}(t) d t
$$

где $f_{k}$ - функция дисконта.

Таким образом, у каждой подотрасли имеется своя функция полезности, зависящая от вида функции дисконта, которая отражает предпочтения по получению прибыли с теченим времени. В некоторых подотраслях важнее получить большую прибыль сейчас, а некоторым, наоборот, важнее большая прибыль в будущем, чем маленькая сейчас.

Функция дисконта всегда убывает, поскольку получение денег в более ранний момент времени всегда ценнее, чем в более поздний. Обозначим коэффициент скорости убывания через

$$
r_{k}(t)=-\left(\ln f_{k}(t)\right)^{\prime}=-\frac{f_{k}^{\prime}(t)}{f_{k}(t)}
$$

и банковский процент через $r$.

На интервале, где $r_{k}(t) \leqslant r$, для фирмы в $k$-й подотрасли целесообразно только копить деньги и класть их на депозит, поскольку это со временем неограниченно увеличивает ее полезность. Если $r_{k}(t) \geqslant r$, может быть целесообразно вложение в производство. Наконец, на период, где $r_{k}(t)=0$, никакие планы не целесообразны, поскольку функцию полезности они не меняют.

Далее полагаем, что $r_{k}=$ const, т.е. $f_{k}(t)=e^{r_{k}} t$ до момента времени $T_{k}$. При $t>T_{k}$ будем полагать $r_{k}(t)=0$.

3.4. Нахождение компромиссного решения. Рассмотрим нестратегическую задачу оптимизации поведения фирм: задается стратегия поведения отрасли в целом, согласующая интересы подотраслей.

Напомним определение компромиссного решения (см. [2]). Оно минимизирует потери наименее удачливого участника, где под потерями понимается отклонение реального выигрыша затрат от максимально возможного. В данном случае компромиссное решение минимизирует превыше-

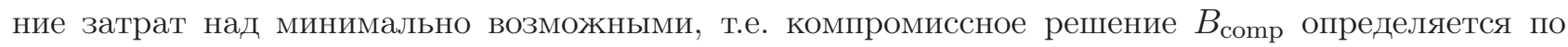
формуле

$$
B_{\text {comp }}=\arg \min _{B} \max _{k}\left(C_{k}(B)-\min _{\tilde{B}} C_{k}(\tilde{B})\right) .
$$

. Компромиссное решение можно найти следующим образом. Сначала для каждого $k$ найдем $B$, минимизирующее $C_{k}(B)$ (обозначим его $B_{k}^{*}$ ) и вычислим для него минимальные затраты $C_{k}^{*}=$ $C_{k}\left(B_{k}^{*}\right)$. Алгоритм их нахождения такой же, как в обычной задаче минимизации затрат, только вместо $r$ нужно подставить $r_{k}$.

Далее нужно найти минимум по $B$ выражения $\max \left(C_{1}(B)-C_{1}^{*}, \ldots, C_{s}(B)-C_{s}^{*}\right)$. Произведя дискретизацию по времени, получим, что нужно найти $n$-мерный вектор $\left(x_{1}, \ldots, x_{n}\right)$, минимизирующий выпуклую функцию (максимум из $s$ выпуклых функций $\left.C_{k}\left(x_{1}, \ldots, x_{n}\right)-C_{k}^{*}\right)$ при наличии линейных ограничений. Этот минимум также можно найти методом условного градиента.

4. Заключение. В данной работе формализована задача оптимальной стратегии покупки оборудования в отрасли. Она решена для важного частного случая, когда не учитывается старение оборудования. Оказывается, что решение при этом может иметь одну из трех форм:

(1) новое оборудование не покупается, используется только уже имеющееся; 
(2) новое оборудование покупается только в начальный момент времени, а далее используется уже имеющееся;

(3) количество покупаемого оборудования постепенно падает в геометрической прогрессии, пока, еще до достижения горизонта планирования, не становится равным нулю.

Для более общей задачи имеет смысл исследовать случаи, когда решением является постоянная функция (покупка оборудования только в начальный момент времени), а также определить скорость сходимости метода условного градиента и решить методом дискретизации времени общую задачу с функцией $U(t, s)$. Это предмет будущих исследований.

Укажем возможности развития модели.

1. Вместо экспоненциальных функций $X(t), Q(t), W(t), f_{k}(t)$ можно взять произвольные функции. Аналогично, старение оборудование и его выход из строя могут происходить не с постоянной скоростью. Соответствующая задача оптимизации при $m=0$, скорее всего, будет обладать свойствами, сходными со свойствами задачи с экспоненциальными функциями. Возможно, что в широком классе случаев можно будет найти минимум методом дискретизации времени и динамического программирования так, что функция $F(t)$ будет кусочно выпуклой. В этом случае аналитическое решение уже невозможно, но возможно численное.

2. Можно рассмотреть функцию Кобба-Дугласа с эффектом масштаба, в которой вместо параметра 1 - a участвует параметр $b$.

3. Можно рассмотреть стратегическую модель: игру с запрещенными ситуациями, в которой разные подотрасли имеют общие ограничения на суммарное количество капитала $K$ и рабочей силы $L$; при этом каждая подотрасль выбирает свою стратегию замены оборудования.

\section{СПИСОК ЛИТЕРАТУРЫ}

1. Беллман Р. Динамическое программирование. - М.: ИЛ, 1960.

2. Малафеев $О$. А., Муравъев А. И. Математические модели конфликтных ситуаций и их разрешение. СПб: СПбГУЭФ, 2000.

3. Ando A. K., Modigliani F., Rashe R., Turnovsky S. J. On the role of expectations of price changes and technological change in an investment function// Int. Econ. Rev. - 1974. - 15, № 2. - P. 384-414.

Малафеев Олег Алексеевич

Санкт-Петербургский государственный университет

E-mail: malaf eyevoa@mail.ru

Парфенов Андрей Павлович

Санкт-Петербургский государственный университет (СПбГУ);

Институт проблем региональной экономики РАН, Санкт-Петербург

E-mail: keldoor@gmail.com 